\title{
Topological Indices of Semigraph
}

\author{
Ambika Biradar
}

\begin{abstract}
Topological indices of different molecular structures has been widely studied since last many decades. These indices are treated as a score function which maps a structure to a real numerical value and this value can be used as a descriptor of the structure which helps to study the structure and under its characteristics. Topological indices are been defined in many ways based on distances between nodes, degree of a node. In this paper we will be discuss various types of topological indices of some special classes of semigraphs. Here we will use the adjacent degree of end vertices to define these indices. Also in this paper we will find the generalised formula of these indices for complete Semigraph $E_{n}^{c}$ and $T_{n-1}^{1}$
\end{abstract}

Keywords: Semigraph, Randic index,Zagreb index.

\section{INTRODUCTION}

The concept of Semigraph was first studied by Sampathkumar E as generalization of graph.[1], [2]

Definition : Semigraph is a pair $S(V, E)$, where $V$ is a non void set of elements called vertices \& $E$ is the set of ordered n-tupples of distinct vertices called edges for $n \geq 2$ where these edges satisfy the following two conditions

1. any of the two edges have atmost one vertex in common

2. any two edges $e=\left(e_{1}, e_{2}, e_{3}, \ldots . . e_{r}\right)$ and

$$
\begin{aligned}
& f=\left(f_{1}, f_{2}, f_{3}, \ldots \ldots . . f_{s}\right) \text { are equal if } \\
& \text { i) } r=s \text { and } \\
& \text { ii) } e_{i}=f_{i} \text { for } 1 \leq i \leq r \text { or } e_{i}=f_{r-i+1}
\end{aligned}
$$

this implies that $e=\left(e_{1}, e_{2}, e_{3}, \ldots . . e_{r}\right)$ is same as $e=\left(e_{r}, e_{r-1}, e_{r-2} \ldots . . e_{1}\right) e_{1}$ and $e_{r}$ are called the end vertices of an edge $e$, while $e_{2}, e_{3}, \ldots \ldots e_{r-1}$ are called the middle vertices of edge $e$.

Cardinality of edge is the number of vertices in an edge and it is denoted as $|e|$.

Adjacent vertices: Two vertices are said to be adjacent if they belong to same edge and they are said to be consecutively adjacent if they belong to same edge and are consecutive in order.

Adjacent edges: Two edges are said to be adjacent if there is a vertex in common.

Revised Manuscript Received on February 05, 2020.

* Correspondence Author

Ambika Biradar*, Department of Mathematics, Dr.D.Y.Patil Institute of Technology,Pimpri-411018.India. Email: Ambika.biradar@dypvp.edu.in

(C) The Authors. Published by Blue Eyes Intelligence Engineering and Sciences Publication (BEIESP). This is an open access article under the CC BY-NC-ND license (http://creativecommons.org/licenses/by-nc-nd/4.0/)
For a vertex $v$ in a semigraph $S(V, E)$ various types of degree are defined as follows

1) $d(v)$ :Degree of vertex $v$ is the number of edges having $v$ as an end vertex.

2) $d_{e}(v)$ :Edge degree of a vertex $v$ is the number of edges containing vertex $v$.

3) $d_{a}(v)$ : Adjacent degree of a vertex $v$ is the number of vertices adjacent to $v$.

4) $d_{c a}(v)$ Consecutive adjacent degree of a vertex $v$ is the number of vertices which are consecutively adjacent to $V$.

Example. 1 Let $S(V, E)$ be a semigraph where

$V=(1,2,3,4,5,6)$

and

$E=(1,3,5),(1,4,6),(2,4,5),(5,6)$

In $S(V, E), 1,2,5,6$ are end vertices, 3 and 4 are middle vertices

$d(1)=2, d(2)=1, d(5)=3, d(6)=2$,

$d_{e}(1)=2, d_{e}(2)=1, d_{e}(5)=3, d_{e}(6)=2$,

$d_{a}(1)=4, d_{a}(2)=2, d_{a}(5)=5, d_{a}(6)=3$,

Special Types of Semigraphs[3]

Regular Semigraph : A semigraph $\mathrm{G}$ is said to be regular if all its vertices have the same degree of a particular given type of degree

Uniform Semigraph : A semigraph $G$ is said to be r-uniform if cardinality of each edge in $\mathrm{G}$ is $\mathrm{r}$.

Complete Semigraph: If any two vertices in a semigraph $\mathrm{S}$ are adjacent then the semigraph $\mathrm{S}$ is said to be complete . Following are some of the families of semigraphs which are complete

1) $E_{r}^{c}$ a semigraph consisting of a single s-edge of cardinality $\mathrm{r}$.

2)) $T_{r-1}^{1}$ a semigraph consisting of an s-edge of cardinality $\mathrm{r}-1$ and one vertex joined with each of the $\mathrm{r}-1$

vertices by an edge of cardinality two.

Incidence matrix and Adjacency matrix of a semigraph has been studied by Y S Gaidhani and C M Deshpande see[2]. In this paper the degree matrix of a Semigraph is defined .

Let $S(V, E)$ be a semigraph with vertex set $V=(1,2,3,4, \ldots . . p)$ and edge set $E=\left(e_{1}, e_{2}, e_{3}, \ldots . . e_{p}\right)$ then degree matrix of semigraph is defined as 
$D(S)=\left\lfloor a_{i j}\right\rfloor$ where

$a_{i j}=d_{a}(i), \quad i$ is an end vertex

$$
=0 \text {, otherwise }
$$

where $d_{a}(i)$ is an adjacent degree of vertex $i$.

$d_{a}(i)=\sum_{i \in e_{j}}\left(\left|e_{j}\right|-1\right)$

Topological index of a structure is a numerical value which quantitates structure and its branching pattern. These indices are used to study quantitative structure property relationship (QSPR) and quantitative structure activity relationship (QSAR). [4]\&[5]

Randic index of a graph $\mathrm{G}(\mathrm{V}, \mathrm{X})$ is defined $R(G)=\sum_{e f \varepsilon X}[d(e) d(f)]^{-\frac{1}{2}}$

It is also known as product connectivity index.

Sum connectivity index $\chi(G)=\sum_{e f \varepsilon X}[d(e)+d(f)]^{\frac{-1}{2}}$

First Zagreb index is defined as $M_{1}(G)=\sum_{v \varepsilon V}(d(v))^{2}$

Second Zagreb index is defined as $M_{2}(G)=\sum_{e f \varepsilon X}(d(e) d(f))$

These topological indices are calculated using degrees of a vertex.

In order to define topological indices for semigraph adjacent degree of end vertex and middle end vertex is considered.

Topological indices of Semigraph

Example 1. $S(V, X), V=(1,2,3,4,5,6,7)$

$X=\left\{e_{1}=(1,2,3,4), e_{2}=(4,5,6), e_{3}=(2,6)\right.$

End vertices are $1,4,6$

Middle end vertex: 2

$$
d_{a}(1)=3, d_{a}(4)=5, d_{a}(6)=3, d_{a}(2)=1,
$$

Randic index $R(G)=\sum_{e f \varepsilon X}[d(e) d(f)]^{-\frac{1}{2}}$

$$
\begin{aligned}
& =\left[d_{a}(1) d_{a}(4)\right]^{\frac{-1}{2}}+\left[d_{a}(4) d_{a}(6)\right]^{\frac{-1}{2}} \\
& +\left[d_{a}(2) d_{a}(6)\right]^{\frac{-1}{2}} \\
& =1.0937
\end{aligned}
$$

\section{Sum connectivity index}

$$
\begin{aligned}
& \chi(G)=\sum_{e f \varepsilon X}[d(e)+d(f)]^{\frac{-1}{2}}= \\
& =\left[d_{a}(1)+d_{a}(4)\right]^{\frac{-1}{2}}+\left[d_{a}(4)+d_{a}(6)\right]^{\frac{-1}{2}} \\
& +\left[d_{a}(2)+d_{a}(6)\right]^{\frac{-1}{2}} \\
& =1.027
\end{aligned}
$$

First Zagreb index $M_{1}(G)=\sum_{v \varepsilon V}(d(v))^{2}$

$=3^{2}+5^{2}+3^{2}+1^{2}=44$

Second Zagreb index $M_{2}(G)=\sum_{e f \varepsilon X}(d(e) d(f))$

$=15+15+3=33$

\section{Topological index for special class of Semigraph}

1] Consider Complete Semigraph $E_{r}^{c}$ with $r$ vertices and a single edge of cardinality $r E_{r}^{c}(V, X)$

$V=(1,2,3,4,5,6,7, \ldots \ldots \ldots . . r)$ and

$X=\left\{e_{1}=(1,2,3,4,5,6, \ldots r)\right\}$

End vertices are 1 and $\mathrm{r}$,

$d_{a}(1)=r-1, d_{a}(r)=r-1$,

Randic index of $R(G)=\sum_{e f \varepsilon X}[d(e) d(f)]^{\frac{-1}{2}}$

$==\left[d_{a}(1) d_{a}(r)\right]^{-\frac{1}{2}}=\frac{1}{r-1}$

Sum connectivity

index

$\chi(G)=\sum_{e f \delta X}[d(e)+d(f)]^{\frac{-1}{2}}$

$=\left[d_{a}(1)+d_{a}(r)\right]^{\frac{-1}{2}}=\frac{1}{\sqrt{2(r-1)}}$

First Zagreb index $M_{1}(G)=\sum_{v \varepsilon V}(d(v))^{2}$

$=(r-1)^{2}+(r-1)^{2}=2(r-1)^{2}$

Second Zagreb index $M_{2}(G)=\sum_{e f \varepsilon X}(d(e) d(f))$

$=(r-1)(r-1)=(r-1)^{2}$

2. Consider complete Semigraph $T_{r-1}^{1}$ consisting of a s-edge of cardinality $(r-1)$ and one vertex joined with each of $(r-1)$ vertices by one edge of cardinality two.

$V=(1,2,3,4,5,6,7, \ldots \ldots \ldots . . r)$

$X=\left\{e_{1}=(1,2,3,4,5,6, \ldots . r-1), e_{2}=(1, r)\right.$,

$\left.e_{3}(2, r) \ldots . . e_{r}(r-1, r)\right\}$

$d_{a}(1)=r-1, d_{a}(r-1)=r-1, d_{a}(r)=r-1$

And $d_{a}(i)=1,2 \leq i \leq r-2$ 
Randic index of $R(G)=\sum_{e f \varepsilon X}[d(e) d(f)]^{\frac{-1}{2}}=$ $\frac{3}{(r-1)}+\frac{r-3}{\sqrt{r-1}}$

Sum connectivity index $\chi(G)=\sum_{e f \varepsilon X}[d(e)+d(f)]^{\frac{-1}{2}}$

$$
=\frac{3}{\sqrt{2(r-1)}}+\frac{r-3}{\sqrt{r}}
$$

First Zagreb index $M_{1}(G)=\sum_{v \varepsilon V}(d(v))^{2}$

$=3(r-1)^{2}+r-3=3 r^{2}-5 r$

Second Zagreb index $M_{2}(G)=\sum_{e f \varepsilon X}(d(e) d(f))$

$=3(r-1)^{2}+(r-3)(r-1)=4 r^{2}-10 r+6$

\section{RESULT ANALYSIS}

For a simple(no loops and multiple edges) connected graph $\mathrm{G}(\mathrm{V}, \mathrm{X})$ where $\mathrm{V}$ is set of vertices and $\mathrm{X}$ is set of edges and $|V|=r$ and $|X|=m$ First Zagreb indices and second Zagreb indices satisfy the following inequality $M_{1}+2 M_{2} \leq 4 m^{2}$ and $M_{1} \geq \frac{4 m^{2}}{r}$ see[6].

\begin{tabular}{|c|c|c|}
\hline $\begin{array}{l}\text { Topological } \\
\text { Indices }\end{array}$ & $E_{r}^{c}$ & $T_{r-1}^{1}$ \\
\hline $\begin{array}{l}\text { First Zagreb index } \\
M_{1}(G)\end{array}$ & $2(r-1)^{2}$ & $3 r^{2}-5 r$ \\
\hline $\begin{array}{l}\text { Second } \quad \text { Zagreb } \\
\text { index } M_{2}(G)\end{array}$ & $(r-1)^{2}$ & $4 r^{2}-10 r+6$ \\
\hline $\begin{array}{ccc}\text { Randic } & \text { index } & \text { of } \\
R(G) & & \end{array}$ & $\frac{1}{r-1}$ & $\frac{3}{(r-1)}+\frac{r-3}{\sqrt{r-1}}$ \\
\hline $\begin{array}{l}\text { Sum connectivity } \\
\text { index } \chi(G)\end{array}$ & $\frac{1}{\sqrt{2(r-1)}}$ & $\frac{3}{\sqrt{2(r-1)}}+\frac{r-3}{\sqrt{r}}$ \\
\hline
\end{tabular}

Here we observe that these inequalities are satisfied by Semigraph $S(V, X)$ also

$$
\begin{aligned}
& M_{1}+2 M_{2} \leq 4 m^{2} \quad, \quad M_{1} \geq \frac{4 m^{2}}{r} \quad \text { where } \\
& m=\sum_{e_{j} \in X}\left(\left|e_{j}\right|-1\right) \text { and } r=|V|
\end{aligned}
$$

\section{CONCLUSION}

In this paper we have obtained generalized formula of various topological indices for special class of semigraph. These results help to understand the topology of the graph networks.

\section{FUTURE SCOPE}

Topological indices can be calculated with the help of eigen values and Laplacian eigenvalues in case of larger structure.

\section{REFERENCES}

1. E.Sampathkumar "Semigraphs and their applications", Report on the DST project, submitted to Department of Science and Technology, India, May-2000

2. Gaidhani Y.S,Deshpande C.M. ,Athawale B.P “Adjacency Matrix of Semigraph",Electronic Notes in Discrete Mathematics, 2017

3. Bam B.Y , .Bhave N.S "On Some problems of Graph Theory in Semigraphs”by, , Ph.D Thesis, University of Pune.

4. B. L. Liu and I. Gutman "Estimating the Zagreb and the general randic indices,", MATCH Communications in Mathematical and in Computer Chemistry, vol. 57, no. 3, pp. 617-632, 2007.

5. B. Zhou and N. Trinajstic, "On general sum-connectivity index," by Journal of Mathematical Chemistry, vol. 47, no. 1, pp. 210-218, 2010

6. Tamás Réti "On the Relationships between the First and Second Zagreb Indices" by,MATCH Communications in Mathematical and in Computer Chemistry · January 2012

\section{AUTHORS PROFILE}

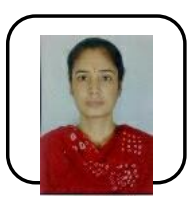

Ambika Biradar, is currently working as Assistant Professor at DIT,Pimpri. She has done her Post graduation in Mathematics from University of Pune. Her area of interest is Graph theory and Lattice theory. 